Check for updates

Cite this: Phys. Chem. Chem. Phys., 2020, 22, 7404

Received 28th January 2020, Accepted 15th March 2020

DOI: $10.1039 / \mathrm{d} 0 \mathrm{cp} 00470 \mathrm{~g}$

rsc.li/pccp

\section{High-resolution resonance-enhanced multiphoton photoelectron circular dichroism $\dagger$}

\author{
Alexander Kastner, (iD a Greta Koumarianou, (D) b Pavle Glodic, (iD b

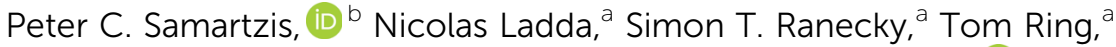 \\ Sudheendran Vasudevan, ${ }^{a}$ Constantin Witte, ${ }^{a}$ Hendrike Braun, (ID a Han-Gyeol Lee, ${ }^{a}$ \\ Arne Senftleben, (iD a Robert Berger, ${ }^{C}$ G. Barratt Park, (D) de Tim Schäfer (DD *de and \\ Thomas Baumert (iD a
}

\begin{abstract}
Photoelectron circular dichroism (PECD) is a highly sensitive enantiospecific spectroscopy for studying chiral molecules in the gas phase using either single-photon ionization or multiphoton ionization. In the short pulse limit investigated with femtosecond lasers, resonance-enhanced multiphoton ionization (REMPI) is rather instantaneous and typically occurs simultaneously via more than one vibrational or electronic intermediate state due to limited frequency resolution. In contrast, vibrational resolution in the REMPI spectrum can be achieved using nanosecond lasers. In this work, we follow the highresolution approach using a tunable narrow-band nanosecond laser to measure REMPI-PECD through distinct vibrational levels in the intermediate $3 s$ and $3 p$ Rydberg states of fenchone. We observe the PECD to be essentially independent of the vibrational level. This behaviour of the chiral sensitivity may pave the way for enantiomer specific molecular identification in multi-component mixtures: one can specifically excite a sharp, vibrationally resolved transition of a distinct molecule to distinguish different chiral species in mixtures.
\end{abstract}

\section{Introduction}

Enantiomer-specific spectroscopy of chiral molecules in the gas phase $^{1}$ is a dynamically evolving research field, where techniques like e.g. microwave three-wave mixing, ${ }^{2-5}$ chiral-sensitive high harmonic generation, ${ }^{6-8}$ controlled enantioselective orientation with an optical centrifuge, ${ }^{9}$ or Coulomb explosion imaging for absolute configuration determination ${ }^{10-14}$ have been developed in recent years. Chiral signatures are also present in both the photoion and photoelectron signal when chiral molecules are ionized with circularly polarized light. Circular dichroism (CD) in the ion yield has been investigated using resonance enhanced multi-photon ionization (REMPI) in combination with mass spectrometry. ${ }^{15-20}$ Coulomb explosion

\footnotetext{
${ }^{a}$ Universität Kassel, Heinrich-Plett-Str. 40, 34132 Kassel, Germany

${ }^{b}$ Institute of Electronic Structure and Lasers, Foundation for Research and Technology - Hellas (FORTH), P. O. Box 1527, 71110 Heraklion, Greece

${ }^{c}$ Fachbereich Chemie, Philipps-Universität Marburg, Hans-Meerwein-Straße 4, 35032 Marburg, Germany

${ }^{d}$ Georg-August-Universität Göttingen, Tammannstr. 6, 37077 Göttingen, Germany. E-mail: tschaef4@gwdg.de

${ }^{e}$ Max Planck Institut für Biophysikalische Chemie, Am Fassberg 11, 37077 Göttingen, Germany

$\dagger$ Electronic supplementary information (ESI) available. See DOI: 10.1039/ d0cp00470g
}

imaging techniques have recently allowed transient chirality to be investigated in the molecular frame. ${ }^{21}$ In contrast to REMPI-based CD of the photoion yield, which arises from weak interference effects between electric and magnetic dipole transition moments, the chiral signature of the photoelectron angular distribution after laser ionization originates from an electric dipole interaction alone. In 1976, it was theoretically predicted that the photoionization of optically active molecules by circularly polarized light would give rise to a substantial forward-backward asymmetry in the angular distribution of the photoelectrons. $^{22}$ This effect, known as photoelectron circular dichroism (PECD), was first demonstrated by single-photon ionization of bromocamphor using a synchrotron source in $2001 .^{23}$ Subsequently, PECD was also demonstrated on other bicyclic ketones using pulsed femtosecond table-top laser systems to drive $2+1$ REMPI. $^{24,25}$ The angular distribution of photoelectrons was measured using velocity map imaging. ${ }^{26-28}$ Multiphoton PECD has also been observed for methyloxirane ${ }^{29}$ and limonene. ${ }^{30,31}$ Because PECD has been observed when using single-photon ionization out of an achiral core-shell initial orbital, $^{32,33}$ it has been interpreted as being largely a final state effect, strongly influenced by the long-range scattering potential. ${ }^{34}$ In contrast, PECD has also been observed for photoelectrons with high kinetic energy above $500 \mathrm{eV}$ resulting from a Fano interference. ${ }^{35}$ PECD is sensitive to the final vibrational 
level of the cation, ${ }^{34,36,37}$ conformation of the molecule, ${ }^{37,38}$ and to subcycle interactions with tailored bichromatic light fields. ${ }^{39,40}$

The underlying electric dipole interaction makes PECD very sensitive to small changes in the amount of enantiomeric excess (e.e.) and consequently the technique shows promise as a highly sensitive analytical tool ${ }^{24,25,41-44}$ exhibiting sub one percent sensitivity. ${ }^{45}$ It is possible to measure enantiomeric excesses in multi-component mixtures by recording massselected PECD spectra with electron image-ion mass coincidence methods. ${ }^{46}$ This approach requires significant experimental effort so that alternative methods are desirable. For instance, PECD in high-resolution REMPI in molecular beams using nanosecond laser systems could be a promising spectroscopic approach as speculated previously by Janssen et $a l^{47}$ The technique permits vibrational resolution, so that different chiral molecules in mixtures can be distinguished by their vibrational features in the REMPI spectra. This has already been demonstrated in REMPI-CD experiments that have taken advantage of high laser resolution to observe sign changes when investigating REMPI-CD via different vibrational levels of the intermediate electronic state ${ }^{48}$ or to distinguish different conformers. ${ }^{49}$ PECD spectra belonging to a certain molecular species can also be recorded by ionizing via narrow, unambiguously assigned REMPI transitions. Of course, for these kinds of experiments rotational and vibrational degrees of freedom should be cooled, which can be efficiently achieved using supersonic molecular beams.

Recently, a femtosecond pump-probe setup was used to observe picosecond dynamics in the 3s Rydberg intermediate state of fenchone, one of the typical benchmark molecules for PECD measurements. ${ }^{50}$ The PECD value was found to vary on a picosecond timescale. However, due to the large frequency bandwidth of the femtosecond laser pulse (30 meV), no vibrational resolution could be achieved. In other studies on fenchone, the dependence on intermediate electronic state and photoelectron kinetic energy has been investigated. ${ }^{31,51}$ This information provides valuable benchmarks for emerging theoretical descriptions of multiphoton PECD, ${ }^{25,52-56}$ and coherent control approaches. ${ }^{57}$

In this contribution we demonstrate partially vibrationally resolved multiphoton PECD of fenchone, obtained by highresolution $2+1$ REMPI via intermediate Rydberg states with a nanosecond pulsed dye laser system. Earlier we observed nanosecond-pulse PECD without vibrational resolution with the help of a frequency tripled Nd:YAG laser. ${ }^{58}$ Experiments are supported by theoretical modeling of the electronically excited states' vibrational level structure. In the low-energy region of the $3 \mathrm{~s}$ state, the features observed in a previous $2+1$ REMPI spectrum ${ }^{51}$ can be assigned to single vibrational eigenstates. As the photon energy is increased, the density of vibrational states increases rapidly and intramolecular vibrational redistribution (IVR) dynamics of the vibrational wavepacket that is excited on the $3 \mathrm{~s}$ Rydberg state become important. We do not observe significantly different PECD values when ionizing fenchone either via a resolved eigenstate or via a wavepacket made up of many strongly interacting vibrational levels. Hence, the vibrational character of the intermediate 3s Rydberg state does not substantially influence the PECD of fenchone after $2+1$ REMPI. This work is the first demonstration of PECD employing tunable dye laser systems providing vibrational resolution. The approach presented here based on high-resolution REMPI spectroscopy has the potential to facilitate enantiomer-specific analytical measurements of multi-component mixtures.

\section{Methods}

\subsection{Experimental setup and data evaluation}

The experimental setup is shown in Fig. 1. A commercial dye laser (Lambda Physik LPD 3000) was pumped by a Lambda Physik LPX 315i XeCl excimer laser (10 Hz, 308 nm, 25 ns). The output of the dye laser was tuned over the wavelength region 375-420 nm using the laser dyes QUI, PBBO, and Exalite 416. The pulse energy was typically 3-6 $\mathrm{mJ}$ and the bandwidth was $0.1 \mathrm{~cm}^{-1}$. The approximate laser wavelength was measured using an Avantes AvaSpec 3648 spectrometer (324-482 nm range, about $0.04 \mathrm{~nm}$ resolution). A more precise calibration was obtained by comparing the peak positions in the REMPI spectrum to those that were measured previously, ${ }^{51}$ which were calibrated to an accuracy of $\pm 0.03 \mathrm{~cm}^{-1}$ using a high-precision wavemeter (HighFinesse, WS7).

The high-resolution spectrum of fenchone ${ }^{51}$ recorded with linearly polarized light shows the partially resolved vibrational structure of 3s and 3p electronic states (shown in blue in Fig. 2). It agrees in the lower wavenumber range nicely with the spectrum reported by Driscoll et al. ${ }^{59}$ who recorded the $3 \mathrm{~s}$ region. Different electronic intermediate states can be populated by the two-photon transition. The two lowest-lying electric dipole-allowed Rydberg transitions lead to electronic intermediates having $3 \mathrm{~s}$ and $3 \mathrm{p}$ Rydberg character. ${ }^{60}$ The transition from these intermediates to the continuum was observed to be governed by a $\Delta v=0$ propensity rule in a previous femtosecond experiment. ${ }^{51}$

The laser beam polarization was initially linear and oriented in the plane of the detector. An achromatic quarter-wave plate (B.Halle, 300-470 $\mathrm{nm}$ achromatic with air gap) was used to convert linearly polarized (LIN) to left circularly polarized (LCP)

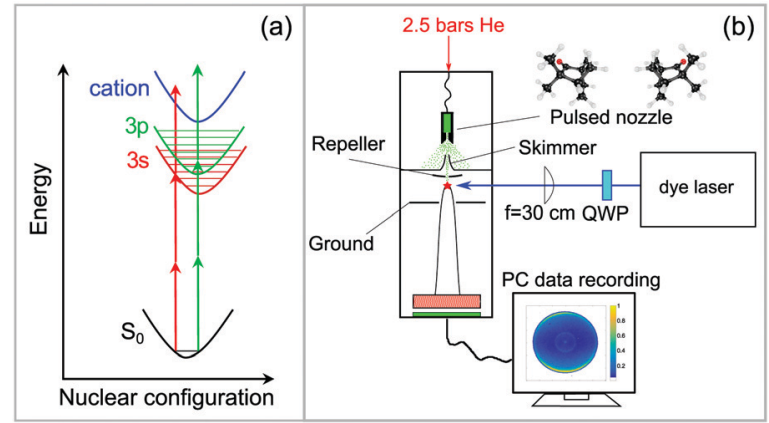

Fig. 1 (a) $2+1$ Resonance-enhanced multiphoton ionization (REMPI) scheme: two photons are necessary to excite vibrational levels of the $3 \mathrm{~s}$ or $3 p$ Rydberg states while another photon ionizes the molecule. (b) Experimental setup for the vibrationally resolved PECD experiment using a high resolution dye laser. A detailed description is given in the text. 


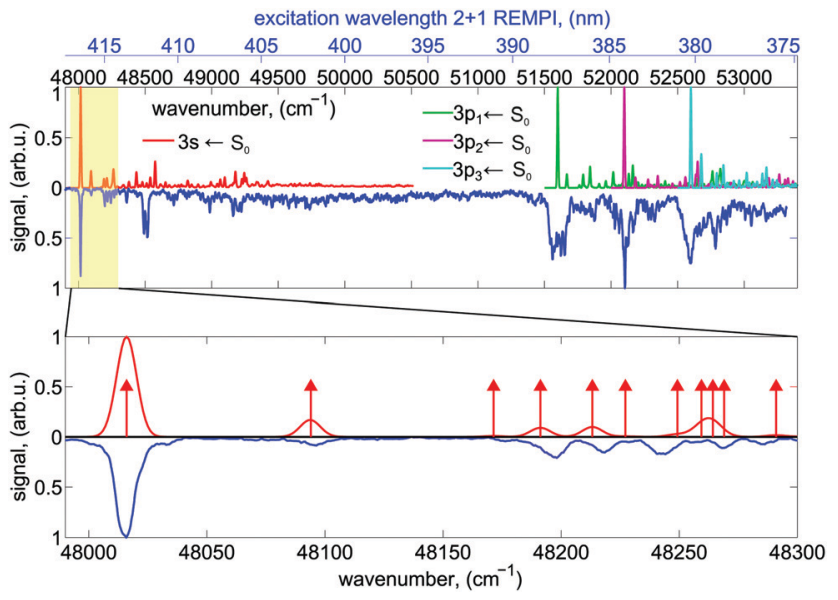

Fig. 2 The experimental $2+1$ REMPI spectrum of the $3 s \leftarrow n$ and $3 p \leftarrow n$ transitions of fenchone recorded with linearly polarized light is shown in blue. For comparison, the calculated Franck-Condon profiles for the transition to the $3 \mathrm{~s}$ state (red) and the transitions to the three components of the $3 p$ state, denoted here by $3 p_{1}, 3 p_{2}$, and $3 p_{3}$ (green, purple, turquoise) are shown. The origin of the individual profiles have been shifted by about $4500 \mathrm{~cm}^{-1}$ (namely $4348 \mathrm{~cm}^{-1}, 4257 \mathrm{~cm}^{-1}, 4466 \mathrm{~cm}^{-1}, 4746 \mathrm{~cm}^{-1}$ for $3 s, 3 p_{1}, 3 p_{2}, 3 p_{3}$, respectively) to higher wavenumbers compared to the computed 0-0 transition wavenumbers. See text for more information. Intensities of the different FC profiles were scaled such that all 0-0 transitions are normalized to the same intensity. In the bottom panel, an enlarged view of the region around the band origin of the $3 \mathrm{~s}$ state, where the vibrational levels are well resolved, is shown. Transitions to individual vibrational eigenstates are indicated by red arrows with their length being unrelated to the Franck-Condon factor.

or right circularly polarized (RCP) light. The wavelength scan was split into three parts corresponding to the dyes used. The quality of polarization was determined using a Glan-Laser polarizer (ThorLabs GL10) and a power meter (Ophir Nova II). The circularity of polarization is expressed via the Stokes $\left|S_{3}\right|$ parameter and has been measured for each dye at different wavelength settings. Except for one measurement point at $396 \mathrm{~nm}$, where the laser power was unstable, $\left|S_{3}\right|$ was well above $96 \%$. No correction of the experimental data with respect to the $\left|S_{3}\right|$ value was performed.

The enantiopure $(S)-(+)$ - and $(R)-(-)$-fenchone samples were purchased from Sigma-Aldrich with a specified purity of $99.2 \%$ (fenchone) and used without further purification. The enantiomeric excess (e.e.) as measured by gas chromatography was $99.9 \%$ for $(S)-(+)$-fenchone and $84 \%$ for $(R)-(-)$-fenchone. ${ }^{45}$ Liquid fenchone, soaked into an inert cotton wad, was evaporated in a heatable sample compartment near the tip of a home-built pulsed solenoid valve nozzle, which is based on the Even-Lavie $\operatorname{design}^{61}$ and has been described in detail previously. ${ }^{62}$ The fenchone sample was heated to about $100{ }^{\circ} \mathrm{C}$, and 2.5 bars of He was used as a backing gas. The supersonic beam was singly skimmed before entering the detection chamber, where it is intersected by the laser beam at a $90^{\circ}$ angle.

An $f=300 \mathrm{~mm}$ plano-convex quartz lens was used to focus the laser beam into the interaction region of an imaging photoelectron spectrometer. The focal distance was chosen to avoid power broadening of the absorption lines. The photoelectron spectrometer, which has been explained in detail in previous work, ${ }^{63}$ uses velocity map-imaging (VMI) as demonstrated by Eppink and Parker ${ }^{27,28}$ with the ion focusing lens included in the geometry of the repeller and ground plates (see Fig. 1b). The VMI provides an energy resolution of about $\Delta E / E \approx 5 \%$. Energy calibration of the VMI was performed using ionization of Xe to the ${ }^{2} P_{1 / 2}$ and ${ }^{2} P_{3 / 2}$ continuum states via a four-photon transition driven by the third harmonic of a Nd:YAG laser. The VMI can be used to project either the photoelectrons or the photoions onto a home-built imaging detector composed of multi-channel plates (BASPIK) with a $50 \mathrm{~mm}$ diameter and a P47 phosphor screen (Proxivision). Three dimensional photoelectron angular distributions (PADs) are projected onto the detector and measured as PAD images using a 1.4 million pixel CCD camera (Unibrain Fire-i 702b). The VMI voltage was set such that photoelectrons with kinetic energy up to about $4.1 \mathrm{eV}$ could be measured. Alternatively, ion TOF mass spectra can be recorded on an oscilloscope (Hameg model 1507-3; $150 \mathrm{MHz}, 200 \mathrm{MS} / \mathrm{s}$ ) via a capacitively coupled output. At each wavelength, photoelectron images for LIN, LCP, and RCP laser light polarization were averaged for 1800 laser pulses ( $\sim 3$ minutes). The PECD image was calculated by subtracting the averaged RCP PAD image from the averaged LCP PAD image.

The original three-dimensional photoelectron distribution is reconstructed using an Abel inversion routine, where two different algorithms have been used. The LIN PADs were evaluated by a polar onion peeling ${ }^{64}$ algorithm written in MATLAB. The polar onion peeling algorithm runs on the pixel grid provided by the camera. The LCP and RCP PADs were evaluated using a C++ based pBasex algorithm, ${ }^{65}$ which was kindly provided by G. A. Garcia from SOLEIL. Details on the evaluation can be found in the Supporting Information. We used a Legendre polynomial basis function set that includes both odd and even order polynomials up to the 6th order following Yang's theorem. ${ }^{66}$ Using the pBasex algorithm, different peaks in the photoelectron spectrum can be evaluated separately. The chiral signature is contained in the odd-order Legendre polynomial coefficients. The magnitude of PECD can be derived by computing a sum over the odd-order coefficients $c_{i}$ normalized to the total signal $c_{0}$, denoted as linear PECD: ${ }^{41}$

$$
\mathrm{LPECD}=\frac{1}{c_{0}}\left(2 c_{1}-\frac{1}{2} c_{3}+\frac{1}{4} c_{5}\right) .
$$

The LPECD value for a measurement is derived using a weighted average over the range of radii contained within the full width at half maximum (FWHM) of the peak signal.

\subsection{Computational methods}

Equilibrium structures and harmonic vibrational frequencies of fenchone in the electronic ground state and various Rydberg states were calculated on the approximate second order coupled cluster level (CC2) using the so-called resolution of the identity (RI) as implemented in the quantum chemistry program Turbomole. ${ }^{67}$ As in some of the calculations reported in previous work, ${ }^{52}$ we have chosen the cc-pVTZ basis set on all 
atoms except oxygen. For the latter we chose the t-aug-cc-pVTZ basis set. For $\mathrm{C}$ and $\mathrm{H}$ we chose the corresponding recommended RI basis sets (cbas, jkbas), for $\mathrm{O}$ we used the basis set recommended for d-aug-cc-pVTZ for fitting of the Coulomb and exchange terms (jkbas) and the original t-aug-cc-pVTZ basis also as RI basis set within the electron correlation treatment (cbas). The latter choice is not ideal, but resulted, in contrast to test calculations with the RI basis recommended for d-aug-ccpVTZ, only in vertical excitation wavenumbers into the relevant Rydberg states that are systematically lower by about $46 \mathrm{~cm}^{-1}$ to $50 \mathrm{~cm}^{-1}$. Resulting structural changes in the electronic ground state were found to be negligible. Electrons in the 11 energetically lowest molecular orbitals, which are composed mostly of atomic 1s orbitals of oxygen and carbon, were kept frozen in the electron correlation treatment (frozen core approximation). Convergence criteria for the self-consistent field (SCF) energies were chosen to be $10^{-9} E_{\mathrm{h}}$. Convergence criteria for the root mean square of the density matrix used in energy minimization and vibrational frequency calculations was on the order of $10^{-8}$. Ground state coupled cluster equations were iterated until the energy change between two cycles remained below $10^{-8} E_{\mathrm{h}}$. The convergence threshold for the norm of residual vectors in subsequent linear response calculations of excitation energies was chosen on the $10^{-6}$ level. Convergence criteria for equilibrium structures were imposed such that the norm of analytic gradients of the energy with respect to displacements of the nuclei remained below $10^{-5} E_{\mathrm{h}} a_{0}^{-1}$ and the estimated energy change between two optimization steps was below $10^{-8} E_{\mathrm{h}}$. Franck-Condon profiles for excitations from the electronic ground state into the various Rydberg states were calculated with a development version of the hotFCHT program ${ }^{68-70}$ using both time-dependent and time-independent methodologies in the harmonic approximation. In the time-dependent approach, a Gaussian lineshape function with a full width at half maximum of $10 \mathrm{~cm}^{-1}$ was chosen to mimic the approximate width of the $0_{0}^{0}$ transition region of the $3 \mathrm{~s}$ state. The timeindependent approach was utilized with pre-screening criteria such that at least $97 \%$ of the total integrated FC profile could be obtained. A maximum of five simultaneously excited normal modes was included for this purpose. The graining in the timeindependent calculations was chosen to be $1 \mathrm{~cm}^{-1}$. The calculated spectra are compared with experiment in Fig. 2.

\section{Results and discussion}

\subsection{Resonance enhanced multiphoton ionization}

The $2+1$ REMPI spectrum recorded with linearly polarized light shown in blue in Fig. 2 probes the excitation via the $3 \mathrm{~s}$ state at energies $>48000 \mathrm{~cm}^{-1}$ and the excitation via the $3 \mathrm{p}$ states at energies $>51500 \mathrm{~cm}^{-1}$. Vibronic transitions are on average well separated below excitation energies of $48300 \mathrm{~cm}^{-1}$. In this region, the nanosecond laser $\left(0.1 \mathrm{~cm}^{-1}\right.$ bandwidth $)$ populates mostly single vibrational eigenstates during the REMPI process.

With increasing wavenumber, however, the vibrational density of states grows rapidly, so that zero-order bright states are excited that can couple to a manifold of dark vibrational states located within the $0.1 \mathrm{~cm}^{-1}$ laser bandwidth leading at higher wavenumbers to IVR probably on a sub-picosecond timescale. ${ }^{71}$ Spectral lines at energies above $51500 \mathrm{~cm}^{-1}$ are significantly broadened indicating a fast dissipation channel out of the $3 p$ Rydberg states. In the plots, the origins of the computed Franck-Condon profiles were shifted by about $4500 \mathrm{~cm}^{-1}$ (namely $4348 \mathrm{~cm}^{-1}, 4257 \mathrm{~cm}^{-1}, 4466 \mathrm{~cm}^{-1}$, $4746 \mathrm{~cm}^{-1}$ for $3 \mathrm{~s}, 3 \mathrm{p}_{1}, 3 \mathrm{p}_{2}, 3 \mathrm{p}_{3}$, respectively) to higher wavenumbers compared to the computed $0_{0}^{0}$ transitions to match the experimentally observed spectrum. This corresponds only to one of several tentative assignments that appear possible for the three components of the $3 p$ Rydberg state. The lowest fundamental vibration at $48090 \mathrm{~cm}^{-1}$ is a delocalized butterfly motion of the ring systems. Vibrations between $48170 \mathrm{~cm}^{-1}$ and $48300 \mathrm{~cm}^{-1}$ are methyl torsional and skeletal modes. More details about the vibrations can be found in the Supporting Information.

We recorded photoelectron images at $27((R)-(-)$-fenchone $)$ and $34((S)-(+)$-fenchone) selected excitation wavelengths between $375 \mathrm{~nm}$ and $420 \mathrm{~nm}$ for LIN, LCP, and RCP laser light. At wavelengths longer than $412 \mathrm{~nm}$, the wavelength was tuned to the center of each resolved spectral feature in the REMPI spectrum. At the employed laser pulse energy no signal was observed off resonance. At wavelengths shorter than $412 \mathrm{~nm}$, where the onset of a continuous unresolved background is observed, PAD images were recorded at $1 \mathrm{~nm}$ intervals. The photoelectron spectrum (PES) obtained for different excitation wavelengths of linearly polarized light are shown in Fig. 3(a). Each PES contains a sharp, intense peak corresponding to ionization from the $3 \mathrm{~s}$ intermediate state. At wavelengths shorter than about $389 \mathrm{~nm}$ a weak second contribution originating from excitation of the $3 p$ intermediate states is observed in the PES. As the wavelength is scanned, the photoelectron peak position shifts by an amount equal to the change in the one-photon energy in agreement with findings in a previous femtosecond experiment. ${ }^{51}$ The scaling of photoelectron energy with excitation wavelength agrees well with $h c / \lambda-\left(\mathrm{IP}-E_{3 \mathrm{~s}, 3 \mathrm{p}}\right)$ (shown as white lines in Fig. 3(a)). IP $=8.5 \mathrm{eV}$ is the adiabatic

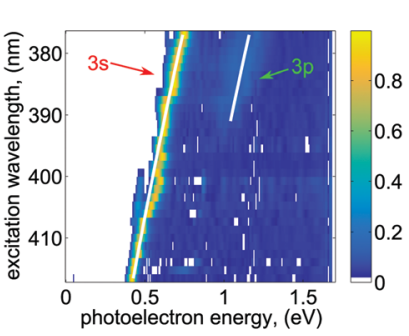

(a)

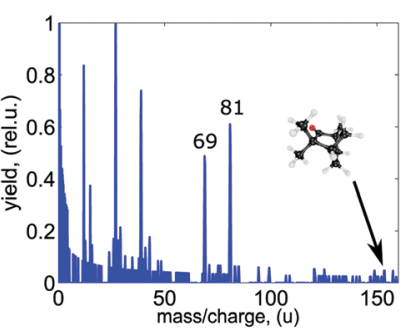

(b)
Fig. 3 (a) Wavelength-dependent PES derived from the LIN PADs for (S)-(+)-fenchone after Abel-inversion using polar onion peeling. ${ }^{64}$ Every row represents a single measurement, where up to two distinct electronic intermediates were observed. The expected energy scaling with $\hbar \omega$ is indicated by the white lines. (b) Mass spectrum for (S)-(+)-fenchone obtained at the $3 \mathrm{~s}$ band origin at $416.57 \mathrm{~nm}$. A detailed discussion can be found in the text. 
ionization energy of fenchone, ${ }^{51,72}$ and $E_{3 \mathrm{~s}}=5.95 \mathrm{eV}$ and $E_{3 \mathrm{p}} \approx$ $6.37 \mathrm{eV}$ are the electronic energies of the $3 \mathrm{~s}$ and $3 \mathrm{p}$ intermediate states. ${ }^{51}$ The photoelectron energy scaling indicates essentially vibrational-energy conserving one-photon ionization out of the intermediate states to the cation, which points to a negligible shift of potentials with respect to structural parameters.

The intensity of the $3 p$ peak relative to the $3 \mathrm{~s}$ peak is significantly weaker than it is in the PES recorded in the same spectral range with femtosecond laser pulse ionization, where the contribution of the $3 p$ state is larger than the contribution of the $3 \mathrm{~s}$ state (see Fig. 5 of ref. 51). However, we observe a sharp increase in the total ion signal of about one order of magnitude when the $3 \mathrm{p}$ excitation threshold is reached. See excitation energies higher than $51500 \mathrm{~cm}^{-1}$ in Fig. 2 and compare to the signal below $51500 \mathrm{~cm}^{-1}$, which corresponds to transitions via the $3 \mathrm{~s}$ state and is non-zero. This observation is consistent with internal conversion (IC) from the $3 p$ state to the $3 \mathrm{~s}$ state, leading to a shortened lifetime of the $3 \mathrm{p}$ state and hence to less ionization out of this state. Instead, molecules are ionized from the $3 \mathrm{~s}$ state and emit photoelectrons carrying the energy signature of the 3 s state (i.e. without the energy that has been converted to vibrational excitation in the $3 \mathrm{~s}$ state). In general IC to the electronic ground state $S_{0}$ or to the energetically low-lying $S_{1}\left(n \rightarrow \pi^{*}\right)$ excited state is also energetically possible. These states would be highly vibrationally excited after IC and are not observed in our experiment probably due to negligible Franck-Condon factors for ionization at the energy that is probed. However, keeping in mind that the total ion signal grows when exciting via the $3 p$ state, and that the photoelectrons carry the energy signature from the 3 s state, our observations suggest that $3 \mathrm{p} \rightarrow 3$ s IC may be much more rapid than $3 \mathrm{p} \rightarrow \mathrm{S}_{0} / \mathrm{S}_{1}$ IC due to the unfavorable Franck-Condon overlap for the latter processes. In contrast, when $2+1$ REMPI via the $3 \mathrm{p}$ state is performed using a femtosecond laser pulse, the timescale of the experiment is too short for $3 p \rightarrow 3 s$ IC to occur, and direct ionization out of the $3 p$ state is observed more frequently. ${ }^{51}$ The intensity of the $3 p$ Rydberg state peak in the photoelectron spectrum is therefore significantly higher than observed in the nanosecond laser experiment.

Pump-probe experiments conducted at $49800 \pm 200 \mathrm{~cm}^{-1}$ have shown that the $3 \mathrm{~s}$ state undergoes internal conversion to the electronic ground state on a timescale of approximately $3.3 \mathrm{ps}^{50}$ The width of the vibrationally resolved lines in the $48000-48500 \mathrm{~cm}^{-1}$ region of our spectrum provides only a lower limit to the lifetime since the rotational structure is not resolved. Assuming a $10 \mathrm{~K}$ rotational temperature, and using the rotational constants reported in ref. 73, we obtain a simulated rotational contour with a FWHM of approximately $5 \mathrm{~cm}^{-1}$, which depends by only a small amount on which component of the rank-two transition moment tensor is chosen. This value is only slightly narrower than the observed $9.1 \mathrm{~cm}^{-1}$ FWHM of the $0_{0}^{0}$ transition. The observed width corresponds to a lower limit of about $0.6 \mathrm{ps}$ for the lifetime. However, the reader should keep in mind that this value is only a lower limit as other phenomena can also lead to spectral line broadening.
The photoionization mass spectrum obtained at the band origin of the $3 \mathrm{~s}$ state at $416.57 \mathrm{~nm}$ is shown in Fig. 3(b). No parent ion is observed and the mass spectrum shows strong fragmentation of the molecules. Prominent fragments with largest mass-to-charge ratio have masses 69 and 81 amu. The observations made here are in agreement with previous nanosecond findings. ${ }^{58}$ It should be noted that the REMPI spectrum presented in Fig. 2 and 5 has been measured in a previous study at significantly reduced laser pulse power and was recorded on the parent ion mass. ${ }^{51}$ An increase in laser intensity leads to increased fragmentation but no change in the PECD as has been observed in a previous fs experiment. ${ }^{41}$ In neither study was the PES or PECD strongly influenced by the fragmentation of the molecules. We thereby conclude that fragmentation of the molecules happens after the ionization in agreement with previous findings. ${ }^{41,58}$ In a recent coincidence experiment on methyloxirane, a link between PECD and the fragmentation channels in strong field ionization has been reported. ${ }^{74}$

\subsection{PECD evaluation}

We calculate the raw PECD image at each wavelength by subtracting the RCP PAD image from the LCP PAD image. ${ }^{41}$ In Fig. 4 we show the antisymmetric part of the raw and Abel-inverted PECD image for the $0_{0}^{0}$ transition at $416.57 \mathrm{~nm}$ and for excitation at $380.2 \mathrm{~nm}$, where ionization via either the $3 \mathrm{~s}$ or $3 \mathrm{p}$ states is possible. We observe a distinct forward/ backward asymmetry of emitted photoelectrons with respect to the propagation axis of light.

From the raw PECD images, we calculate LPECD values for ionization via the 3s Rydberg state at each selected wavelength

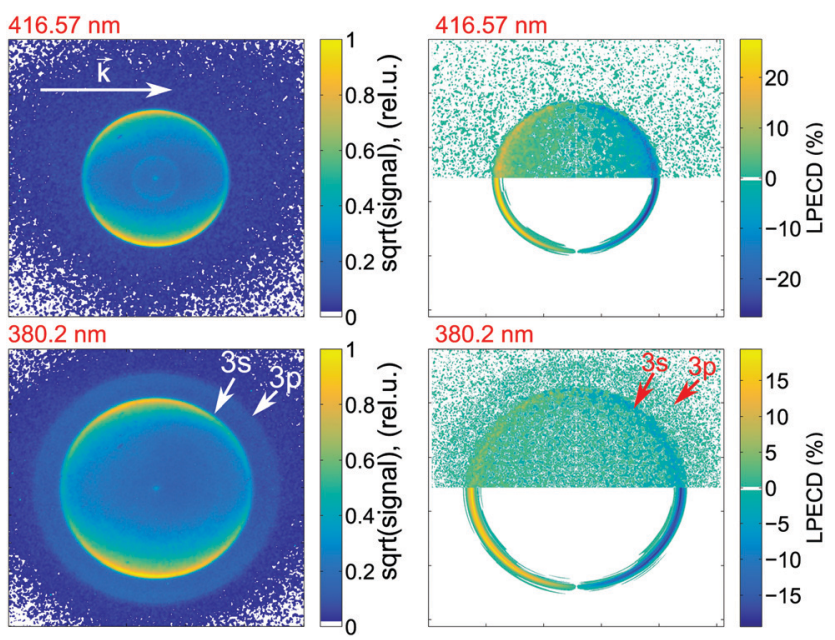

Fig. 4 Photoelectron velocity map images for (S)-(+)-fenchone ionized via $2+1$ REMPI via the $0_{0}^{0}$ transition to the 3 s state using $416.57 \mathrm{~nm}$ light (top row) and via transitions to both the $3 \mathrm{~s}$ and $3 p$ states using $380.2 \mathrm{~nm}$ light (bottom row). The left-hand column shows the photoelectron images obtained using LIN and the right-hand column shows the antisymmetrized PECD images. In the upper half of each PECD image, the raw data is shown and in the lower half the Abel-inverted image is shown. In the images obtained with $380.2 \mathrm{~nm}$ light, the kinetic energy of the intense inner ring and weak outer ring correspond to vertical ionization from the $3 \mathrm{~s}$ and $3 p$ Rydberg states, respectively. The white vector shows the direction of the excitation laser beam. 
as described in Section 2.1. The results for both enantiomers are shown in Fig. 5 alongside the high-resolution REMPI spectrum of fenchone. Within the error of our experiment we observe the same magnitude (but opposite sign) for the two enantiomers taking into account the previously determined ${ }^{45}$ enantiomeric purity of $84 \%$ of $(R)-(-)$-fenchone. The LPECD decreases slightly in magnitude at shorter wavelengths. We could not extract reliable LPECD values for ionization from the 3 p Rydberg state due to poor signal-to-noise ratio (see Fig. 4). Therefore, we were unable to conclude whether the sign change of the LPECD value when ionizing from 3p Rydberg states observed in the femtosecond laser experiments ${ }^{51}$ also happens in the nanosecond laser experiments. In the ESI $\dagger$ we also show the contributions of $c_{1}, c_{3}$, and $c_{5}$ to the LPECD value normalized to $c_{0}$ following eqn (1). The main contribution originates from $c_{1}$.

The LPECD values recorded after nanosecond laser ionization are larger in magnitude than the LPECD values recorded after femtosecond laser ionization ${ }^{51}$ over the measured energy range. The wavelength-dependent LPECD curves of the two measurements exhibit the same shape but are shifted to one another by an LPECD value of $2.5 \%$. However, it should be noted that the femtosecond laser LPECD values were recorded using an effusive beam source, whereas the nanosecond laser LPECD values were recorded using a pulsed molecular beam. Hence, vibrational excitation in the electronic ground state was

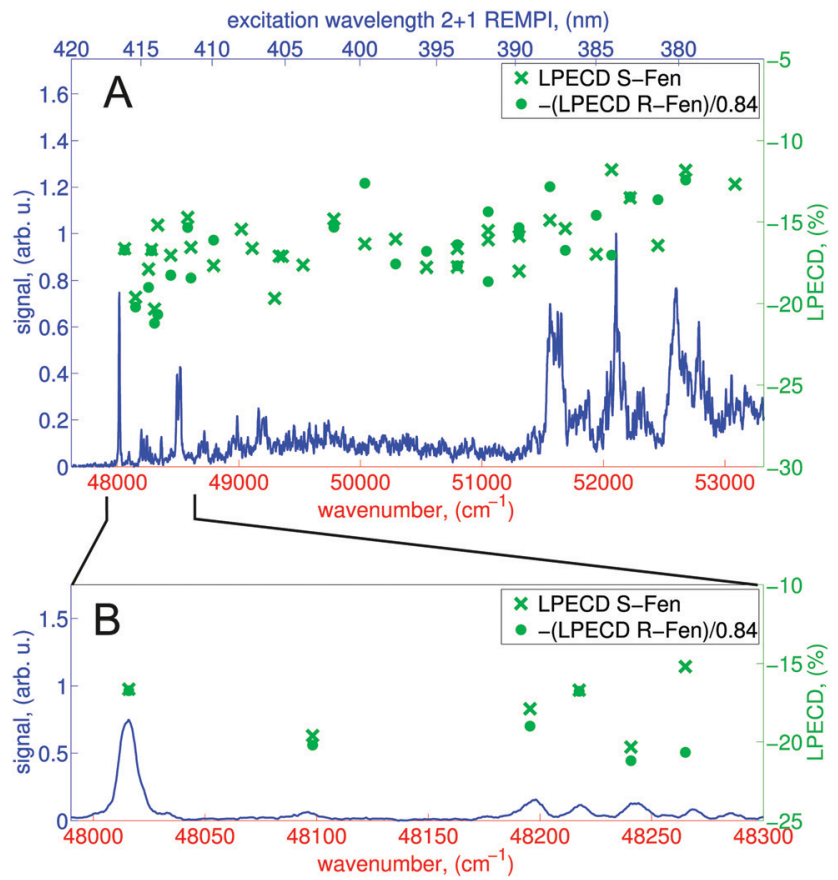

Fig. 5 (A) High-resolution $2+1$ REMPI spectrum showing partially resolved vibrational structure in the intermediate state (blue curve) and LPECD values for both enantiomers (green circles and crosses) for the $3 \mathrm{~s}$ Rydberg state. Based on multiple measurements taken at a fixed frequency we deduce a relative experimental error of $\pm 10 \%$. The blue $y$-axis on the left side refers to the REMPI spectrum. The green $y$-axis on the right side refers to the LPECD values of $(S)-(+)$-fenchone and $(R)-(-)$-fenchone. As indicated in the legend, the LPECD values of $(R)-(-)$-fenchone have been multiplied by $-\frac{1}{0.84}$. (B) Enlarged view of the $3 \mathrm{~s}$ band origin. greater in the femtosecond laser experiment and caution should be taken in making a direct comparison of the results. To make such a comparison, future experiments should use the same molecular beam source to identify differences caused only by the excitation laser pulse duration.

As described in the previous section, we distinguish different regions of the spectrum in which the molecular dynamics are different. First, at wavelengths between $416.57 \mathrm{~nm}$ and $412 \mathrm{~nm}$ the density of vibrational levels in the $3 \mathrm{~s}$ state is low so that we ionize fenchone essentially out of a true vibrational eigenstate. Second, at wavelengths between $412 \mathrm{~nm}$ and $388 \mathrm{~nm}$, the density of states (DOS) is high, so that we ionize molecules whose vibrational energy is redistributed over a large number of coupled vibrational levels of the $3 \mathrm{~s}$ state after IVR. Mode selective IC to the electronic ground state could also influence the vibrational state distribution. ${ }^{50}$ Third, at wavelengths shorter than $388 \mathrm{~nm}$ we excite both the $3 \mathrm{~s}$ and the $3 p$ states. However, internal conversion via $3 p$ dominates the mechanism for ionization via $3 \mathrm{~s}$. With increasing wavenumber, however, the vibrational density of states grows rapidly. Hence, the character of the high-lying $3 \mathrm{~s}$ vibrational states that are accessed in this region is no longer governed by FranckCondon overlap with the $\mathrm{S}_{0}$ ground state, but rather by vibronic overlap with low-lying bright vibrational states of $3 \mathrm{p}$.

When comparing the LPECD values in the distinct spectral regions described above, we do not observe any abrupt changes of LPECD values. Furthermore, we do not observe any pronounced features in the LPECD values that correspond with resolved vibrational bands in the REMPI spectrum at the $3 \mathrm{~s}$ origin between $416.57 \mathrm{~nm}$ and $412 \mathrm{~nm}$. The LPECD value varies by $c a . \pm 3 \%$ (absolute) when ionizing via different vibrational states, which is at the level of the experimental error of this measurement.

We therefore conclude that the vibrational energy of the intermediate 3s Rydberg state does not significantly influence the PECD of fenchone after $2+1$ REMPI. This perhaps reflects the Rydberg character of the intermediate electronic state. The electron's probability density is located relatively far from the molecular frame prior to ionization. The movement of the nuclei might therefore have only minor influence on the outgoing electron. This could be advantageous for future experiments as vibrational levels of the intermediate Rydberg state can be treated as spectator states, which do not influence the photoelectron angular distribution or energy in experiments on a nanosecond timescale. However, further experiments on different chiral molecules need to be done to clarify whether these observations can be generalized.

\section{Summary and conclusion}

We used nanosecond laser pulses to obtain the photoelectron circular dichroism (PECD) of the chiral molecule fenchone after $2+1$ resonance enhanced multi-photon ionization (REMPI) via the $3 \mathrm{~s}$ and $3 p$ Rydberg states for selected ionization energies in the spectral range between $375 \mathrm{~nm}$ and $420 \mathrm{~nm}$. In contrast to previous studies using femtosecond laser ionization, we resolve 
vibrational structure in the REMPI spectrum, and assign LPECD values to distinct intermediate vibrational levels of the 3s Rydberg state. We do not observe any strong dependence of the LPECD value on the vibrational level of the intermediate state, which might be a consequence of its Rydberg electronic character. An accompanying computational study identifies the different vibrational modes that give rise to the peaks observed in the $2+1$ REMPI spectrum. We demonstrate the feasibility of PECD measurements with tabletop nanosecond dye laser systems, which can be used to investigate chiral mixtures via high resolution REMPI assisted PECD measurements.

\section{Conflicts of interest}

There are no conflicts to declare.

\section{Acknowledgements}

The authors are grateful to Theofanis Kitsopoulos for support and feedback, and thank G. A. Garcia for sharing the algorithm. Funded by the Deutsche Forschungsgemeinschaft (DFG, German Research Foundation) - Projektnummer 328961117 SFB 1319 and ELCH. A. K., G. B. P. and T. S. acknowledge financial support within the LaserLab Europe network (Grant Agreement No. 654148). T. S. acknowledges support by the DFG under grant INST186/1302-1. P. S. gratefully acknowledges support by HELLAS-CH (MIS 5002735) implemented under "Action for Strengthening Research and Innovation Infrastructures”, funded by the Operational Programme "Competitiveness, Entrepreneurship and Innovation" (NSRF 2014-2020) and co-financed by Greece and the European Union (European Regional Development Fund). Open Access funding provided by the Max Planck Society. R. B. acknowledges the Center for Scientific Computing (CSC) Frankfurt for providing computer time.

\section{Notes and references}

1 Chiral Recognition in the Gas Phase, ed. A. Zehnacker, CRC Press, Boca Raton, 2010.

2 E. Hirota, Proc. Jpn. Acad., Ser. B, 2012, 88, 120-128.

3 D. Patterson and J. M. Doyle, Phys. Rev. Lett., 2013, 111, 23008.

4 D. Patterson, M. Schnell and J. Doyle, Nature, 2013, 497, 475-477.

5 S. Eibenberger, J. Doyle and D. Patterson, Phys. Rev. Lett., 2017, 118, 123002.

6 A. E. Cireasa, R. Boguslavskiy, B. Pons, M. C. H. Wong, D. Descamps, S. Petit, H. Ruf, N. Thiré, A. Ferré, J. Suarez, J. Higuet, B. E. Schmidt, A. F. Alharbi, F. Légaré, V. Blanchet, B. Fabre, S. Patchkovskii, O. Smirnova, Y. Mairesse and V. R. Bhardwaj, Nat. Phys., 2015, 11, 654.

7 D. Baykusheva and H. J. Wörner, Phys. Rev. X, 2018, 8, 031060.

8 O. Neufeld, D. Ayuso, P. Decleva, M. Y. Ivanov, O. Smirnova and O. Cohen, Phys. Rev. X, 2019, 9, 031002.
9 A. A. Milner, J. A. M. Fordyce, I. MacPhail-Bartley, W. Wasserman, V. Milner, I. Tutunnikov and I. S. Averbukh, Phys. Rev. Lett., 2019, 122, 223201.

10 M. Pitzer, M. Kunitski, A. S. Johnson, T. Jahnke, H. Sann, F. Sturm, L. P. H. Schmidt, H. Schmidt-Böcking, R. Dörner, J. Stohner, J. Kiedrowski, M. Reggelin, S. Marquardt, A. Schießer, R. Berger and M. S. Schöffler, Science, 2013, 341, 1096-1100.

11 P. Herwig, K. Zawatzky, M. Grieser, O. Heber, B. JordonThaden, C. Krantz, O. Novotny, R. Repnow, V. Schurig, D. Schwalm, Z. Vager, A. Wolf, O. Trapp and H. Kreckel, Science, 2013, 342, 1084-1086.

12 M. Pitzer, G. Kastirke, P. Burzynski, M. Weller, D. Metz, J. Neff, M. Waitz, F. Trinter, L. P. H. Schmidt, J. B. Williams, T. Jahnke, H. Schmidt-Böcking, R. Berger, R. Dörner and M. Schöffler, J. Phys. B: At., Mol. Opt. Phys., 2016, 49, 234001.

13 M. Pitzer, G. Kastirke, M. Kunitski, T. Jahnke, T. Bauer, C. Goihl, F. Trinter, C. Schober, K. Henrichs, J. Becht, S. Zeller, H. Gassert, M. Waitz, A. Kuhlins, H. Sann, F. Sturm, F. Wiegandt, R. Wallauer, L. P. H. Schmidt, A. S. Johnson, M. Mazenauer, B. Spenger, S. Marquardt, S. Marquardt, H. Schmidt-Bocking, J. Stohner, R. Dorner, M. Schoffler and R. Berger, ChemPhysChem, 2016, 17, 2465-2472.

14 M. Pitzer, R. Berger, J. Stohner, R. Dörner and M. Schöffler, Chimia, 2018, 72, 384-388.

15 U. Boesl von Grafenstein and A. Bornschlegl, ChemPhysChem, 2006, 7, 2085-2087.

16 R. Li, R. Sullivan, W. Al-Basheer, R. M. Pagni and R. N. Compton, J. Chem. Phys., 2006, 125, 144304.

17 H. G. Breunig, G. Urbasch, P. Horsch, J. Cordes, U. Koert and K.-M. Weitzel, ChemPhysChem, 2009, 10, 1199-1202.

18 J. Lepelmeier, K. Titze, A. Kartouzian, U. Boesl and U. Heiz, ChemPhysChem, 2016, 17, 4052.

19 U. Boesl, A. Bornschlegl, C. Logé and K. Titze, Anal. Bioanal. Chem., 2013, 405, 6913-6924.

20 P. Horsch, G. Urbasch and K.-M. Weitzel, Chirality, 2012, 24, 684-690.

21 K. Fehre, S. Eckart, M. Kunitski, M. Pitzer, S. Zeller, C. Janke, D. Trabert, J. Rist, M. Weller, A. Hartung, L. P. H. Schmidt, T. Jahnke, R. Berger, R. Dörner and M. S. Schöffler, Sci. Adv., 2018, 5, 7923.

22 B. Ritchie, Phys. Rev. A: At., Mol., Opt. Phys., 1976, 13, 1411-1415.

23 N. Böwering, T. Lischke, B. Schmidtke, N. Müller, T. Khalil and U. Heinzmann, Phys. Rev. Lett., 2001, 86, 1187-1190.

24 C. Lux, M. Wollenhaupt, T. Bolze, Q. Liang, J. Köhler, C. Sarpe and T. Baumert, Angew. Chem., Int. Ed., 2012, 51, 5001-5005.

25 C. S. Lehmann, N. Bhargava Ram, I. Powis and M. H. M. Janssen, J. Chem. Phys., 2013, 139, 234307.

26 D. W. Chandler and P. L. Houston, J. Chem. Phys., 1987, 87, 1445-1447.

27 A. T. J. B. Eppink and D. H. Parker, Rev. Sci. Instrum., 1997, 68, 3477-3484.

28 D. W. Chandler, P. L. Houston and D. H. Parker, J. Chem. Phys., 2017, 147, 013601. 
29 M. M. R. Fanood, I. Powis and M. M. H. Janssen, J. Phys. Chem. A, 2014, 118, 11541-11546.

30 M. M. Rafiee Fanood, M. H. M. Janssen and I. Powis, Phys. Chem. Chem. Phys., 2015, 17, 8614-8617.

31 S. Beaulieu, A. Ferré, R. Géneaux, R. Canonge, D. Descamps, B. Fabre, N. Fedorov, F. Légaré, S. Petit, T. Ruchon, V. Blanchet, Y. Mairesse and B. Pons, New J. Phys., 2016, 18, 102002.

32 U. Hergenhahn, E. E. Rennie, O. Kugeler, S. Marburger, T. Lischke, I. Powis and G. Garcia, J. Chem. Phys., 2004, 120, 4553-4556.

33 V. Ulrich, S. Barth, S. Joshi, U. Hergenhahn, E. Mikajlo, C. J. Harding and I. Powis, J. Phys. Chem. A, 2008, 112, 3544-3549.

34 G. A. Garcia, L. Nahon, S. Daly and I. Powis, Nat. Commun., 2013, 4, 2132.

35 G. Hartmann, M. Ilchen, P. Schmidt, C. Küstner-Wetekam, C. Ozga, F. Scholz, J. Buck, F. Trinter, J. Viefhaus, A. Ehresmann, M. S. Schöffler, A. Knie and P. V. Demekhin, Phys. Rev. Lett., 2019, 123, 043202.

36 G. A. Garcia, H. Dossmann, L. Nahon, S. Daly and I. Powis, ChemPhysChem, 2017, 18, 500-512.

37 M. M. Rafiee Fanood, H. Ganjitabar, G. A. Garcia, L. Nahon, S. Turchini and I. Powis, ChemPhysChem, 2018, 19, 921-933.

38 S. Turchini, J. Phys.: Condens. Matter, 2017, 29, 503001.

39 P. V. Demekhin, A. N. Artemyev, A. Kastner and T. Baumert, Phys. Rev. Lett., 2018, 121, 253201.

40 S. Rozen, A. Comby, E. Bloch, S. Beauvarlet, D. Descamps, B. Fabre, S. Petit, V. Blanchet, B. Pons, N. Dudovich and Y. Mairesse, Phys. Rev. X, 2019, 9, 031004.

41 C. Lux, M. Wollenhaupt, C. Sarpe and T. Baumert, ChemPhysChem, 2015, 16, 115-137.

42 M. H. M. Janssen and I. Powis, Spectroscopy Online, 2017, 15, 16-23.

43 L. Nahon, L. Nag, G. A. Garcia, I. Myrgorodska, U. Meierhenrich, S. Beaulieu, V. Wanie, V. Blanchet, R. Géneaux and I. Powis, Phys. Chem. Chem. Phys., 2016, 18, 12696-12706.

44 A. Comby, E. Bloch, C. M. M. Bond, D. Descamps, J. Miles, S. Petit, S. Rozen, J. B. Greenwood, V. Blanchet and Y. Mairesse, Nat. Commun., 2018, 9, 5212.

45 A. Kastner, C. Lux, T. Ring, S. Züllighoven, C. Sarpe, A. Senftleben and T. Baumert, ChemPhysChem, 2016, 17, 1119-1122.

46 M. M. Rafiee Fanood, N. Bhargava Ram, C. S. Lehmann, I. Powis and M. H. M. Janssen, Nat. Commun., 2015, 6, 7511.

47 M. H. M. Janssen and I. Powis, Phys. Chem. Chem. Phys., 2014, 16, 856-871.

48 J. Lepelmeier, J. L. Alonso-Gómez, F. Mortaheb, U. Boesl, U. Heiz and A. Kartouzian, Phys. Chem. Chem. Phys., 2017, 19, 21297-21303.

49 A. Hong, C. M. Choi, H. J. Eun, C. Jeong, J. Heo and N. J. Kim, Angew. Chem., Int. Ed., 2014, 53, 7805-7808.
50 A. Comby, S. Beaulieu, M. Boggio-Pasqua, D. Descamps, F. Legare, L. Nahon, S. Petit, B. Pons, B. Fabre, Y. Mairesse and V. Blanchet, J. Phys. Chem. Lett., 2016, 7, 4514-4519.

51 A. Kastner, T. Ring, B. C. Krüger, G. B. Park, T. Schäfer, A. Senftleben and T. Baumert, J. Chem. Phys., 2017, 147, 013926.

52 R. E. Goetz, T. A. Isaev, B. Nikoobakht, R. Berger and C. P. Koch, J. Chem. Phys., 2017, 146, 24306.

53 I. Dreissigacker and M. Lein, Phys. Rev. A: At., Mol., Opt. Phys., 2014, 89, 053406.

54 A. N. Artemyev, A. D. Müller, D. Hochstuhl and P. V. Demekhin, J. Chem. Phys., 2015, 142, 244105.

55 A. D. Müller, A. N. Artemyev and P. V. Demekhin, J. Chem. Phys., 2018, 148, 214307.

56 A. D. Müller, E. Kutscher, A. N. Artemyev and P. V. Demekhin, J. Chem. Phys., 2020, 152, 044302.

57 R. E. Goetz, C. P. Koch and L. Greenman, Phys. Rev. Lett., 2018, 122, 013204.

58 A. Kastner, T. Ring, H. Braun, A. Senftleben and T. Baumert, ChemPhysChem, 2019, 20, 1416-1419.

59 J. Driscoll, T. Baer and T. J. Cornish, J. Mol. Struct., 1991, 249, 95-107.

60 F. Pulm, J. Schram, S. Grimme and S. Peyerimhoff, Chem. Phys., 1997, 224, 143-155.

61 U. Even, J. Jortner, D. Noy, N. Lavie and C. Cossart-Magos, J. Chem. Phys., 2000, 112, 8068-8071.

62 G. B. Park, B. C. Krüger, S. Meyer, D. Schwarzer and T. Schäfer, J. Chem. Phys., 2016, 144, 194308.

63 V. Papadakis and T. N. Kitsopoulos, Rev. Sci. Instrum., 2006, 77, 083101.

64 G. M. Roberts, J. L. Nixon, J. Lecointre, E. Wrede and J. R. R. Verlet, Rev. Sci. Instrum., 2009, 80, 053104.

65 G. A. Garcia, L. Nahon and I. Powis, Rev. Sci. Instrum., 2004, 75, 4989-4996.

66 C. N. Yang, Phys. Rev., 1948, 74, 764-772.

67 TURBOMOLE V7.3 2018, a development of University of Karlsruhe and Forschungszentrum Karlsruhe $\mathrm{GmbH}$, 1989-2007, TURBOMOLE GmbH, since 2007, available from http://www.turbomole.com.

68 R. Berger, C. Fischer and M. Klessinger, J. Phys. Chem. A, 1998, 102, 7157-7167.

69 H.-C. Jankowiak, J. L. Stuber and R. Berger, J. Chem. Phys., 2007, 127, 234101.

70 J. Huh and R. Berger, J. Phys.: Conf. Ser., 2012, 380, 012019.

71 D. J. Nesbitt and R. W. Field, J. Phys. Chem., 1996, 100, 12735-12756.

72 D. C. Frost, N. P. C. Westwood and N. H. Werstiuk, Can. J. Chem., 1980, 58, 1659-1665.

73 D. Loru, M. A. Bermúdez and M. E. Sanz, J. Chem. Phys., 2016, 145, 074311.

74 K. Fehre, S. Eckart, M. Kunitski, C. Janke, D. Trabert, J. Rist, M. Weller, A. Hartung, L. P. H. Schmidt, T. Jahnke, R. Dörner and M. Schöffler, J. Phys. Chem. A, 2019, 123, 6491-6495. 